\title{
BAUDELAIRE Y LA FANTASMAGORÍA DEL FIN
}

\author{
Marcelo Jacques de Moraes \\ Universidad Federal de Río de Janeiro \\ Consejo Nacional de Desarrollo Científico y Tecnológico \\ mjdemoraes@gmail.com
}

Traducción de Luciana di Leone

Resumen: La obra poética y crítica de Charles Baudelaire se instituye sobre la tensión entre el imaginario de las "fantasmagorías" (Walter Benjamin) que asalta el cotidiano citadino moderno y la imaginación poética que lucha por captarlo como "esbozo", como figura - aunque sea apenas de paso ("Un éclair... puis la nuit!") - que se disponga al recuerdo, como "memoria del presente" (Le peintre de la vie moderne).

Sin embargo, lo que tal vez constituya sobre todas las cosas la singularidad de Baudelaire -su modo propio de "despertar" - es la consciencia de que el trabajo del poeta encontrará su finalidad $-y$, en cierto sentido, su límite y su fin - en la puesta en escena (más que en la superación o en el enfrentamiento) de ese impasse entre la fascinación hipnótica de un mundo que ya no puede ofrecerse sino como espectáculo, con su potencia permanente de olvido y el deseo de convertirlo en "experiencia".

Este artículo investiga algunas inflexiones del problema, haciendo énfasis en el modo como la obra de Baudelaire ofrece rasgos interesantes para el debate contemporáneo sobre el fin del mundo - y de la poesía.

Palabras clave: Baudelaire, Benjamin, Modernidad, Fin, Fantasmagoría.
Abstract: The poetic and critical work of Charles Baudelaire is instituted on the tension between the imaginary of the "phantasmagoria" (Walter Benjamin) that assaults the daily city life and the poetic imagination that struggles to catch it as an "outline", as a figure - even if it is just in passing ("Un éclair ... puis la nuit!") - which is available to remember, as "memory of the present" (Le peintre de la vie moderne).

However, what perhaps constitutes Baudelaire's uniqueness - his own way of "awakening" - is the awareness that the work of the poet will find its purpose - and, in a certain sense, its limit and its end - in the staging (more than in the overcoming or in the confrontation) of that impasse between the hypnotic fascination of a world that can no longer be offered but as a spectacle, with its permanent power of oblivion and the desire to turn it into "experience".

This article investigates some inflections of the problem, emphasizing how Baudelaire's work offers interesting features for the contemporary debate about the end of the world - and poetry.

Keywords: Baudelaire, Benjamin, Modernity, End, Phantasmagoria. 
En su conferencia de apertura del coloquio "El fin, el resto y el comienzo: coloquio sobre lo contemporáneo", realizado en Río de Janeiro en 2013, João Camillo Penna evocaba el "veredicto del fin" a partir del que se constituyó y se consumó la modernidad occidental. ${ }^{1}$ Entramos en el siglo XX y lo atravesamos discutiendo el problema del fin a partir de varias narrativas y de varios puntos de vista: nos hemos referido de forma intermitente, aunque cada vez con más frecuencia, al fin de la filosofía, al fin de la historia, al fin del arte, al fin de la poesía, etc. Y, frente a la "expansión indeterminada de un modelo homogéneo y homogeneizante, sin bordes", que pasó a dominar ampliamente las condiciones de producción en todos los dominios de la vida, en función de la aceleración cada vez más desmedida y, de cierta forma, auto-administrada de la diseminación planetaria de la tecnología y de las catástrofes más o menos localizadas que irrumpieron un poco en todos los lugares, a partir de esa omnipresencia, frente a ese estado de cosas, surgimos en el siglo XXI todavía, y tal vez más que nunca, bajo el signo de la espera del fin. Espera cada vez más anunciada, pero también cada vez más desarticulada e impotente, sino melancólica, o cínica: claramente, la catástrofe política y económica global, ya amplia e incesantemente notificada en las últimas décadas, y la catástrofe ambiental, para muchos en estado avanzado e irreversible. El fin del mundo, en fin...

\footnotetext{
1 Coloquio realizado en la Facultad de Letras de la Universidad Federal do Rio de Janeiro en noviembre de 2013. Disponible en: https://www.youtube.com/watch?v=5FGarnZVSU. Acceso: 22 jun. 2017.
}

$N^{\circ} 8$. Primer Semestre de 2018 
Para enfrentar ese diagnóstico que, a pesar de los matices de tono, está bastante extendido en el pensamiento contemporáneo y que, en última instancia, parece relacionarse con esa gran fantasmagoría moderna que es la ideología del progreso, lo que nos cabe, como sugería Penna, sería intentar pensar las condiciones del "fin del fin", del "tiempo de la posteridad [la nuestra, naturalmente] que empieza por el fin". Se trataría, por lo tanto, según me parece, de intentar pensar en contrapunto crítico al proceso de totalización que subyace a lo moderno - las condiciones de lo que adviene, o podría advenir, como potencia de lo que nos es propiamente contemporáneo, y como comienzo de otros tiempos, como lo que comienza por el fin, y que tal vez no sea, aún, es lo que esperamos, el fin del mundo, sino el fin de un mundo.

Lo que especialmente me interesa recoger de esa reflexión, para tomarla como punto de partida de la mía, es la idea de que aún vivimos acechados por el fantasma - o por la fantasmagoría - del fin: ya que no se puede postular el comienzo o el recomienzo, a no ser en contraposición a un fin, a un más allá de un fin, a no ser a partir de los restos, o de los síntomas, de un mundo que no cesa de acabar, o de no acabar, y de los esbozos de un mundo que no cesa de comenzar, o de no comenzar. Porque, como nos recuerda el poeta y filósofo Michel Deguy en su libro La fin dans le monde, de 2009, "fin" significa al mismo tiempo "cese" ["cessation"] y "finalidad" ["but"], o sea, interrupción y destinación. De hecho, Deguy abre el libro remitiéndonos a la ambigüedad fundamental del "rasgo deseante" presente en la expresión francesa "en finir 
avec", ambigüedad que vale también para el "acabar con" de la lengua portuguesa. Dice Deguy:

La expresión francesa "en finir (avec)" es opaca; [...]. El deseo y el voto de "no acabar con" lo que acabó, y de "acabar con" lo que no acaba, se deja oír o (pre)sentir en su uso. (Deguy, 2009, p. 7)

En seguida, Deguy formula sintéticamente la paradoja de este "duelo" entre lo acabado y lo inacabado, lo finito y lo infinito ["ce duel, du fini et de l'infini"], duelo que, dice, constituye el "secreto de toda la historia" (p. 11). La formulación que quiero dejar como epígrafe para la reflexión que propongo, y que no deja de tener una resonancia benjaminiana, es la siguiente: "ce qui est fini ne fait que commencer" (p. 8) [lo que acabó, recién comienza]".

Es justamente en torno de la discusión sobre las tensiones entre lo acabado y lo inacabado, lo finito y lo infinito, y sobre sus relaciones con la apertura, o reapertura, de la historia (relaciones que, como sabemos, constituyen, tanto para Walter Benjamin como para Charles Baudelaire, un aspecto esencial de la modernidad), que me gustaría retomar aquí el tema del "fin" a partir del proyecto baudelairiano, atravesado, claramente, por una lectura de Benjamin, para ver de qué manera aún nos permite interrogar nuestra relación con nuestra propia contemporaneidad, con lo que en ella, para nosotros, puede "estar apenas comenzando".

Si, como decíamos, en la perspectiva contemporánea del fin del fin, esperamos, o deseamos, darle fin al pasado, a esos restos del pasado que proliferan y se acomodan de manera cada vez 
más vertiginosa y fragmentaria en nuestra experiencia del cotidiano; si esperamos, o deseamos, apropiarnos de esos restos en el presente, recogiéndolos e interpelándolos y tal vez así llevándolos a reabrirse como comienzo; si es esa nuestra expectativa, o nuestro deseo, ella parece de hecho querer contraponerse a cierta perspectiva de lo moderno señalada por Benjamin. Porque, en el caso de lo moderno tal como Benjamin lo piensa, la "novedad" se ofrece a la experiencia en primer lugar como "eterno retorno de lo mismo" (Benjamin, 1989, p. 156), como su "repetición infernal" (cf. Benjamin, 2006, p. 1124-1127) o, llevado al extremo, como la propia imposibilidad del fin. Tal vez porque el pensamiento jamás podría concebir el mundo más allá de la "actualidad eternizada", según la expresión acuñada por Auguste Blanqui en L'éternité par les astres, de 1872, y que Benjamin volvió célebre en la "Conclusión" de 1939 a "París, capital del siglo XIX". Refiriéndose, como explica Benjamin, a "una imagen del progreso que [...] se revela como la fantasmagoría de la propia historia", el pasaje de Blanqui citado enseguida por el filósofo termina así:

Lo que llamamos progreso está encerrado en cada tierra y desaparece con ella. Siempre y en todos lados, en el campo terrestre, el mismo drama, el mismo cuadro, en el mismo estrecho escenario, una humanidad bulliciosa, enfatuada de su grandeza, creyéndose ser el universo y viviendo en su prisión como en una inmensidad, para desaparecer enseguida junto con el planeta, que cargó con el desprecio más profundo el fardo de su orgullo. La misma monotonía, la misma 
inmovilidad en los astros extranjeros. El universo se repite sin fin y patina en el mismo lugar. (2006, pp. 66-67)

En relación con esto, sería imposible no mencionar la "fusée" ("proyectil" o "cohete") de los Diarios intimos de Baudelaire, escrita probablemente en 1861, y citada algunas veces por Benjamin tanto en el libro de los Pasajes como en una conferencia de 1939 sobre "Los cuadros parisinos", a la que me referiré más adelante. Recordemos algunos fragmentos de la fusée baudelairiana:

El mundo va a acabarse. La única razón que tendría para perdurar es que ya existe [...]. Le pido a todo hombre que piensa que me muestre qué subsiste de la vida. [...] ¡Puede que esos tiempos estén muy próximos; quién sabe si ya no han llegado y si el espesamiento de nuestra naturaleza no es el único obstáculo que nos impide apreciar el medio en que respiramos!

Y en cuanto a mí, que dentro de mí siento algunas veces el ridículo de un profeta, sé que jamás encontraré la caridad de un médico. Perdido en este mundo vil, atropellado por la multitud, soy como un hombre agotado, cuyo ojo no ve hacia atrás, en los profundos años, más que desengaño y amargura, y, hacia adelante, más que una tempestad sin nada nuevo, sin enseñanzas ni dolor. Cuando en la noche le roba al destino algunas horas de placer, acunado en su digestión, olvidado - dentro de lo posible - del pasado, satisfecho con su presente y resignado con el futuro, embriagado de su sangre fría y su dandysmo, orgulloso de no ser tan bajo como aquellos que pasan, ese hombre se dice a sí mismo, contemplando el humo de su cigarro: ¡Qué importa adónde van estas conciencias!

Creo que he derivado en lo que la gente del oficio llama un hors d'cuvre. Sin embargo, dejaré estas páginas - porque quiero fechar mi cólera. 
tristeza.

Le monde va finir. La seule raison pour laquelle il pourrait durer, c'est qu'il existe. [...] Je demande à tout homme qui pense de me montrer ce qui subsiste de la vie. [...]

[...] Ces temps sont peut-être bien proches; qui sait même s'ils ne sont pas venus, et si l'épaississement de notre nature n'est pas le seul obstacle qui nous empêche d'apprécier le milieu dans lequel nous respirons!

Quant à moi, qui sens quelquefois en moi le ridicule d'un prophète, je sais que je n'y trouverai jamais la charité d'un médecin. Perdu dans ce vilain monde, coudoyé par les foules, je suis comme un homme lassé dont l'ail ne voit en arrière, dans les années profondes, que désabusement et amertume, et devant lui, qu'un orage où rien de neuf n'est contenu, ni enseignement, ni douleur. Le soir où cet homme a volé à la destinée quelques heures de plaisir, bercé dans sa digestion, oublieux - autant que possible - du passé, content du présent et résigné à l'avenir, enivré de son sang-froid et de son dandysme, er de n'être pas aussi bas que ceux qui passent, il se dit, en contemplant la fumée de son cigare: Que m'importe où vont ces consciences? Je crois que j'ai dérivé dans ce que les gens du métier appellent un hors-d'œuvre. Cependant, je laisserai ces pages, - parce que je veux dater ma colère.

(Baudelaire, 1975, I, pp. 665-667)

tristesse

Al retratarse, de ese modo, como quien vive entre el incurable y solitario desamparo del "profeta" del fin del mundo, que "jamás encuentra la caridad de un médico", y la "embriaguez" "orgullosa" del dandy, "satisfecho con su presente y resignado con el futuro", ocupado solamente con el "humo de su cigarro", Baudelaire actualiza con ambigüedad y auto-ironía la imagen de la "fatuidad" moderna, que ya nos había ofrecido en su reflexión sobre la Exposición universal de 
1855 al prever las consecuencias inevitables de la "idea grotesca" de progreso. Escribía en aquella ocasión:

[...] y las razas disminuidas, si esa exasperante locura durara mucho tiempo, dormirán sobre la almohada de la fatalidad en el sueño rabioso de la decrepitud. Esa fatuidad es el diagnóstico de una decadencia ya demasiado visible. (Baudelaire, 1976, II, p. 580)

El filósofo y crítico André Hirt asoció ese fragmento a la famosa carta de Baudelaire a Manet, de 1865, donde el poeta le dice al pintor que lo considera como "el primero en la decrepitud de su arte" (Baudelaire, 1973, I, p. 397). Es claro que el comentario no puede comprenderse si no señalamos, como lo hace el crítico André Guyaux, "toda la ambigüedad del sentimiento de Baudelaire frente a lo que se acaba o a lo que comienza, o recomienza, en el arte, por ejemplo" (Guyaux, 2007, p.110). Pero veamos la reflexión de Hirt en torno del sentido de esa decrepitud:

Sobre todo, lo que se revela es que el agotamiento es el fondo y el motor de la historia. [...] La historia no se sustenta en algo real por su propia realidad. En suma, su contenido real es vacío. $\mathrm{Y}$ es en ese sentido que se dirige hacia su fin descubriendo tal vez - iprogresivamente! - los círculos de su vacío (Hirt, 2010, p.59).

Como ejemplo de esa marcha humana "progresiva" que se realiza por entre "los círculos de [lo] vacío de la historia", podríamos citar dos poemas que constituyen alegorías realmente fantasmagóricas, verdaderas fantasmalegorías, si me permiten el neologismo. En primer lugar, "Cada uno con su 
quimera" [Chacun sa chimère], poema en prosa de Spleen de París. En él, un cortejo de hombres camina sin rumbo, "curvados" bajo el peso de una "Quimera" que no saben nombrar, movidos apenas "por una invencible voluntad de andar", "con el aspecto resignado de los condenados a esperar siempre". Esos hombres despiertan inicialmente la curiosidad algo contenida del poeta, pero este, como el dandi de la profecía, sucumbe rápidamente a su propia Quimera, a su "irresistible Indiferencia" (Baudelaire, 1975, I, pp. 282-283). A seguir, tenemos un segundo cortejo, el de los "Siete viejos" [Sept vieillards], poema en el que, recordemos, al poeta se le "aparecen" siete "monstruos horrorosos", rumbo a un "fin desconocido", siete viejos que, "a pesar de tanta decrepitud", "parecían eternos". En "París, capital del siglo XIX", Benjamin define este poema como "una fantasmagoría angustiante $[\ldots]$ en el corazón de la flânerie". Cito el pasaje, bastante conocido:

Se trata, en el poema, de la aparición siete veces reiterada de un viejo de aspecto repugnante. El individuo que es presentado de tal modo en su multiplicación, como siempre el mismo, testimonia la angustia del ciudadano de ya no poder, a pesar de la expresión de las singularidades más excéntricas, romper el círculo mágico del tipo. Baudelaire califica el aspecto de esa procesión como infernal. Pero lo nuevo que él persiguió durante toda su vida no está hecho de otra materia a no ser de esa fantasmagoría de lo "siempre igual" (Benjamin, 2006, p.62).

En este sentido, tal vez sea posible especular un poco más en relación a lo que estaba en juego en aquel triunfo que se pone en escena en "El mundo va a acabarse": el triunfo de la repetición infernal, sin "nada nuevo, ni enseñanza ni dolor", 
triunfo sobre los "hombres que piensan", sobre aquellos a quienes cabría "mostrar" lo que "subsiste de la vida" en un mundo regido por esa "fantasmagoría del progreso, que implica la tiranía de lo "siempre-igual"... ¿Qué se necesitaba para sobrevivir en un mundo que "[iba] a acabarse", que tal vez inclusive ya se hubiera acabado, pero que parecía determinado a "perdurar" para siempre? O, citando la fórmula con que Benjamin definió el "heroísmo en Baudelaire", ¿qué se necesitaba para continuar "viviendo en el corazón de la irrealidad" (Benjamin, 1989, p.165)?

Para el poeta parecía no haber otra salida sino exponer la fuerza de auto-exposición de un mundo feérico que prosperaba y permanecía idéntico a sí mismo gracias, precisamente, a su metamorfosis permanente y a su propia evanescencia, celebrada en lo cotidiano por lo que Giorgio Agamben nombrará en sus Estancias como una "epifanía de lo inaprensible" (Agamben, 1998, p.75). Discutiendo la lógica de lo efímero y del fetichismo con Baudelaire y Marx, Hirt parece resumir bien el problema:

La cosa no tiene más que ser, no es posible dejarla ser; ella debe, por medio de su metamorfosis, gracias al apoyo de su ornamentación feérica, entrar en la danza de los objetos evanescentes, en un mundo él también evanescente, iluminado apenas en eclipse por los relámpagos de lo pasajero (Hirt, 1998, p.10).

Un poema ejemplar a este respecto es "El amor a la mentira" [L'Amour du mensonge], que Benjamin asocia "a la extinción de la ilusión". En ese poema Baudelaire expone la metamorfosis de la apariencia en verdad fulgurante en la 
verdad del vacío, que el poema transforma de algún modo en acontecimiento. Sus versos ponen en escena de forma inequívoca, según me parece, el impasse en que se vuelven indisociables la imaginación alegórica y el imaginario de la fantasmagoría -en este sentido me refería a la fantasmalegoría -, impasse modulado en el poema, no casualmente, a través de la imagen de la belleza ilusoria, "extrañamente fresca" de una cortesana "madura", teñida por la "luz de gas" - y que tal vez enmascara (el lector no pueda dejar de desconfiarlo) los indicios de su "decrepitud". Escuchemos el final del poema:

Cuando te veo pasar, mi querida indolente, Al canto de los instrumentos que se quiebra en el cielo raso, Suspendiendo tu andar armonioso y lento, Y paseando el tedio de tu mirar profundo;

Cuando contemplo, bajo la luz de gas que la colorea, Tu pálida frente, embellecida por la mórbida atracción, Donde las antorchas de la noche se encienden como una aurora, $\mathrm{Y}$ tus ojos atraen como los de un retrato,

Pienso: ¡Cómo es bella! ¡Y extrañamente fresca! [...]

Sé que hay ojos, de los más melancólicos, Que no esconden sus secretos preciosos; Bellos estuches sin joyas, medallones sin reliquias, ¡Más vacíos, más profundos que vosotros mismos, oh, Cielos!

¿Pero no basta con que seas apariencia, para alegrar un corazón que huye de la verdad? ¿Qué importan tu estupidez o tu indiferencia? Máscara o adorno, ¡salud! ¡Adoro tu belleza!

Quand je te vois passer, ô ma chère indolente, 
Au chant des instruments qui se brise au plafond

Suspendant ton allure harmonieuse et lente,

Et promenant l'ennui de ton regard profond;

Quand je contemple, aux feux du gaz qui le colore,

Ton front pâle, embelli par un morbide attrait,

Où les torches du soir allument une aurore,

Et tes yeux attirants comme ceux d'un portrait,

Je me dis: Qu'elle est belle! et bizarrement fraîche! [...]

Je sais qu'il est des yeux, des plus mélancoliques,

Qui ne recèlent point de secrets précieux;

Beaux écrins sans joyaux, médaillons sans reliques,

Plus vides, plus profonds que vous-mêmes, ô Cieux!

Mais ne suffit-il pas que tu sois l'apparence,

Pour réjouir un ccur qui fuit la vérité?

Qu'importe ta bêtise ou ton indifférence?

Masque ou décor, salut! J'adore ta beauté.

(Baudelaire, 1975, I, pp. 98-99)

Podríamos destacar lo que aquí nos interesa a la luz de lo propuesto por Hirt sobre las relaciones entre Baudelaire y la fantasmagoría: mientras esta "[oblitera] el carácter artificialmente construido y producido de aquello que promueve", el poema le opone no "una naturaleza sino otro artificio, el de la alegoría: una deformación alegórica contra una transformación ilusoria del mundo" (Hirt, 1998, p.58). Hirt se inspira aquí, claramente, en el propio Benjamin que, en el siguiente fragmento de Parque Central, sintetiza el problema y, según creo, el poema:

$N^{\circ} 8$. Primer Semestre de 2018 
El ambiente objetivo del hombre adopta, cada vez de modo más brutal, la fisionomía de la mercadería. Al mismo tiempo, la propaganda se propone a ofuscar el carácter mercantil de las cosas. A la engañadora transfiguración del mundo de las mercaderías se contrapone su desfiguración en lo alegórico. La mercadería procura verse a sí misma en la cara, verse a sí misma en el rostro. Festeja su humanización en la prostituta. (Benjamin, 1989, p.163)

Creo que son pocas las imágenes que pueden hacernos sentir tan intensamente el "lirismo en el auge del capitalismo" tanto como esa belleza de tinte melancólico de la prostituta del poema, pura apariencia, acabada por la necesidad permanente del "adorno" y de la "máscara" para re-exponerse como mercadería. Al resistir, en su melancolía, a la mirada y al sujeto que la objetifican, la mirada de la vieja cortesana objetifica, a su vez, esa mirada y ese sujeto. En este sentido, en un pasaje de "El pintor de la vida moderna" en el que retoma de modo un poco más ácido los términos de "Amor a la mentira", al referirse a la mujer moderna, Baudelaire convierte a la presa en "predador": "Ella dirige la mirada al horizonte, como el animal predador: la misma exaltación, la misma distracción indolente y también, a veces, la misma rigidez de atención" (Baudelaire, 1975, I, pp. 719-720).

Se vive, así, en un eterno presente en el que sólo se renuevan las apariencias, y sólo por medio de ellas se le puede "robar al destino algunas horas de placer", como quería el poeta en su profecía del fin del mundo. Sucesión de acontecimientos sin acontecimiento, lo que ese presente pone en escena, en última instancia, es siempre la imposibilidad 
absoluta de morir, y, por lo tanto, de otra vida en la que, justamente, "subsistiría la vida", como, por ejemplo, en el penúltimo poema de Flores del mal, "Sueño de un curioso" [Rêve d'un curieux], en el que el poeta relata un sueño en el que iría a presenciar su propia muerte en un teatro. He aquí el fin del poema:

Yo era como el niño ávido por el espectáculo, Odiando el telón como se odia un obstáculo...

$\mathrm{Al}$ fin la fría verdad se reveló:

Yo había muerto sin sorpresa, y la terrible aurora

Me envolvía. - ¡Pero cómo! ¿Eso es todo?

El telón había caído y yo aún esperaba.

J'étais comme l'enfant avide du spectacle,

Haïssant le rideau comme on hait un obstacle...

Enfin la vérité froide se révéla:

J'étais mort sans surprise, et la terrible aurore

M'enveloppait. - Eh quoi! n'est-ce donc que cela?

La toile était levée et $j$ 'attendais encore.

(pp. 438-439)

En un comentario de 1939 sobre "El mundo va a acabarse", Benjamin destacó el modo en que su propia generación ya se había vuelto insensible a ese proceso de mercantilización del mundo, el modo en que estaba completamente alienada en relación al propio "automatismo estrictamente regulado", a su propia "inhumanidad" - expresiones de Benjamin para aquellos síntomas que Baudelaire había señalado en 1860 y que 
ya por entonces lo "desesperaban". He aquí las palabras con que Benjamin termina su conferencia:

La desesperación fue el rescate de esa sensibilidad que, habiendo sido la primera a abordar la gran ciudad, fue la primera a ser sorprendida por un escalofrío [frisson] que nosotros, frente a las múltiples amenazas, demasiado precisas, ya siquiera sabemos sentir. (Benjamin, 2013, p.1018)

De allí podemos desprender hasta qué punto la ambigüedad de Baudelaire siempre fue cara para Benjamin, que veía la multiplicación y el agravamiento de las "amenazas" "precisas" a la vida impuestos cotidianamente como un testimonio de aquella alienación, de la muerte en vida, que el poeta, justamente, profetizaba - aunque sin dejar de sostener la que continúa siendo una cuestión vibrante y, de cierto modo, nuestra: ¿cómo reconocer, más allá de la constante amenaza del fin que nos acecha, algún comienzo - algún "nuevo escalofrío", como ya dijera Victor Hugo sobre Baudelaire, y que Benjamin hace resonar en el pasaje que acabo de citar? ¿Cómo morir de hecho para poder abrirse hacia una nueva vida que no sea la de la repetición infernal, para poder abrirse a lo "desconocido", a lo "nuevo", como exclama el poeta al final de sus Flores del mal?

Ahora bien, como vimos, desde el punto de vista de la fantasmalegoría baudelairiana del fin del mundo, la certeza del fin, la violencia del futuro inevitable y ya de cierto modo consumado, no implicaba una insensibilidad en relación a ciertas modulaciones intensas e inventivas de su presente. La 
experiencia de la belleza de la prostituta, por ejemplo, a pesar de su "pasajereidad", de su carácter "ordinario", o por causa de ellos, tan sólo "concentraba", por mediación del arte, "el sabor amargo o embriagante del vino de la Vida", como decía el poeta de los dibujos de Constantin Guys (Baudelaire, 1976, II, p.724).

Son justamente esas modulaciones las que constituyen la "modernidad" del presente a los ojos de Baudelaire, son justamente esas modulaciones las que lo "fechan", que le dan una fecha concreta a ese presente y que nos permiten, por nuestra parte, fecharlo. Así como la expresión "dar fin" que mencioné al comienzo con Deguy, "fechar" significa no solo, y de manera ambigua, hacer cesar, dejar para atrás aquello que se "fecha", sino también inscribirlo en la historia y así "escribir" la historia en el sentido benjaminiano, esto es, ofrecerlo al futuro como cita, lo que desvirtúa necesariamente su sentido original. Evoco aquí, al mismo tiempo, como se puede percibir, dos tesis famosas de Benjamin sobre la historia que vienen en secuencia en el "Manuscrito N" del Libro de los pasajes: "Escribir la historia significa darle a las fechas su fisionomía"; "Escribir la historia significa [...] citar la historia" (Benjamin, 2006, p.518).

En este sentido, aquellas páginas proféticas en que Baudelaire anunciaba el fin del mundo, aquel "bors d'cuvre" que "fechaba" de algún modo una "cólera", o una "tristeza" - que "fechaba" de algún modo cierto rechazo del presente -, aquel "bors d'cuvre" realiza, como dice Hirt, la "antinomia del fin de la creación" (Hirt, 2010, p.76), instaurando, a partir de ese "fechar", otra temporalidad, en la que justamente lo que se $N^{\circ}$ 8. Primer Semestre de 2018 
ofrece en el presente como "aperitivo" o como "superfluo" dos posibilidades de traducir el "hors d'cuvre" con el que el poeta califica irónicamente su "deriva" -, o lo que se ofrece en el presente como "bors d'auvre", decíamos, solicita del futuro su extracción crítica. En este sentido, la reflexión de Hirt sobre la fecha de la profecía en Baudelaire parece certera:

Una fecha [...] es el relieve del tiempo, el momento en que se sale de su eje para ofrecer su contenido. Porque el tiempo solamente se contiene a sí mismo en totalidad, como un pedazo o un bloque de tiempo, en todas sus dimensiones de presente, pasado y futuro, cuando se bifurca de su puntualidad formada por cada presente en la línea cronológica. Entonces, se abraza a sí mismo, se cava y se estrella en un acontecimiento que barre todo el espectro de su contenido. [...] Es por esa razón que se puede afirmar que lo que caracteriza una fecha es su inacabamiento, su promesa y su profecía (p.68).

Creo finalmente que podríamos decir, a partir de esta reapertura de la constelación Baudelaire-Benjamin, que el fin, el cese y la destinación de aquello que no termina de no acabarse, que no termina de no recomenzar, depende siempre de la extracción crítica de una "cólera", de una "tristeza", de un rechazo, como decíamos; o sea, de nuestra capacidad de redecir esa cólera y esa tristeza, de re-fecharlas, de reactivarlas en pro de lo que intuimos aquí o allí como aquello que "recién comienza". Y, a partir de ahí, darle una fecha a nuestro propio rechazo de nuestro tiempo, nuestra propia profecía del fin del mundo, de nuestro mundo, dándole su "fisionomía", y reescribiendo, reabriendo, nuestra propia historia, la de nuestro 
presente. Se trata, en fin, de una apuesta en relación a nuestra propia sobrevivencia, nuestra propia vida póstuma.

No utilicé la palabra "apuesta" de modo casual. Me gustaría concluir esta "reapertura" con la referencia a una experiencia específica de rechazo de la realidad que fascinó - y fechó - de forma especial al siglo XIX, experiencia a la que ni Baudelaire ni Benjamin fueron insensibles. Experiencia que, tal vez, más que nunca, en el siglo XXI, nos fascine y nos feche, aunque seguramente de otro modo: la experiencia del "jugador".

En su abordaje del juego en el ensayo "Sobre algunos temas baudelairianos", Benjamin compara el jugador contemporáneo con el obrero de fábrica; ambos "[viviendo como] autómatas", sometidos a la "repetición rigurosa": "ambas ocupaciones están igualmente privadas de contenido" (Benjamin, 1989, pp. 127-128). Benjamin evoca en seguida el poema "El juego", esa otra fantasmalegoría infernal de Baudelaire, que termina de este modo:

He ahí el negro cuadro que en un sueño nocturno

Vi desdoblarse bajo mi mirada clarividente.

Yo mismo, en un rincón del antro taciturno,

Me recosté, frío, mudo, envidiando;

Envidiándole a esas personas la pasión tenaz, A esas viejas putas la fúnebre alegría, ¡Y todos gallardos, traficando bajo mi rostro, Uno, su viejo pudor; otra, su belleza!

Y mi corazón se asustó al envidiar tanta pobre gente, Corriendo con fervor al ávido abismo, ¡Y que, borrachos de su propia sangre, preferirían en suma El dolor a la muerte y el infierno a la nada! 
$V$ oilà le noir tableau qu'en un rêve nocturne

Je vis se dérouler sous mon cil clairvoyant.

Moi-même, dans un coin de l'antre taciturne,

Je me vis accoudé, froid, muet, enviant,

Enviant de ces gens la passion tenace,

De ces vieilles putains la funèbre gaieté,

Et tous gaillardement trafiquant à ma face,

L'un de son vieil honneur, l'autre de sa beaute!!

Et mon cour s'effraya d'envier maint pauvre homme

Courant avec ferveur à l'abîme béant,

Et qui, soûl de son sang, préférerait en somme

La douleur à la mort et l'enfer au néant!

(Baudelaire, 1975, I, pp. 95-96)

En su comentario, Benjamin resalta especialmente el hecho de que, en el poema, "el poeta no participa del juego", que "rechaza el estupefaciente con el que los jugadores procuran embotar el consciente, que los hizo vulnerables a la marcha de las agujas del reloj" (Benjamin, 1989, p. 130). Desde esta perspectiva, tendríamos apenas el aspecto negativo del juego de azar, ligado a la "avidez" (p. 128) alienada del jugador, siempre "inclinado hacia uno de los objetos más miserables que existen, el dinero" (2006, p. 530). Esta sería, en relación al juego, "la naturaleza de la vivencia que Baudelaire [habría elevado] a la categoría de verdadera experiencia" (p. 145), para retomar la célebre fórmula de Benjamin al final de ese ensayo.

Pero, a este respecto, me gustaría considerar también el modo más ambivalente con el que, en el Libro de los pasajes, 
Benjamin había definido al "jugador afortunado", en oposición al "supersticioso", como aquel que "reaccionará [a ciertos guiños] incluso antes de percibirlos", aquel cuyo "reflejo motor provocado por el azar" se anticipa a la "conciencia [de] aquello que está por venir", aquel que "apenas cercena [el] futuro que no penetró como tal en su consciencia”. Es, me parece, esa "presencia de espíritu" "adivinadora" (Benjamin, 2006, pp. 553-554) que lleva al jugador experimentado a la acción, es la que causa "envidia" al poeta figurado en el poema de Baudelaire, quien, en compensación, es el único que posee una "mirada clarividente". Esa oposición que se traza en el poema entre la "fría" "clarividencia" del poeta que logra mantener distancia en aquel "antro taciturno", y la "pasión tenaz", el "fervor", del jugador que no puede "evitar" "correr" hacia el "hiante abismo" podría ser elucidada con el siguiente pasaje de "Calle de mano única" sobre la "vidente" Madame Ariane, donde la "videncia", justamente, es asociada a la acción y confrontada a la interpretación:

Signos premonitorios, presentimientos, señales cruzan día y noche nuestro organismo como olas que baten. Interpretarlos o utilizarlos, he ahí la cuestión. Ambas acciones son inconciliables. [...] Porque antes de que tal profecía o aviso sea algo alcanzable, palabra o imagen, su mejor fuerza ya está muerta, la fuerza con la que acierta en nuestro centro y nos obliga, mal sabemos cómo, a actuar de acuerdo con ella. Si dejamos de hacerlo, entonces, y sólo entonces, es posible descifrarla. La leemos. Pero es demasiado tarde. (Benjamin, 1994, pp. 63-64)

Todo está ahí, en ese intervalo entre el instante en que se está listo para "transformar la amenaza del futuro en el ahora $N^{\circ}$ 8. Primer Semestre de 2018 
pleno" - que es la "obra de la corpórea presencia de espíritu", como apunta Benjamin un poco después en ese mismo texto -, entre esa especie de, por decirlo de algún modo, pasaje al acto de ese hombre atento y "valiente" que es el jugador, y el "demasiado tarde" - ese "demasiado tarde" que tantas veces retorna en Baudelaire- del "descifrar" "clarividente" de aquello que pasa, de aquello que se pasa, descifrado del pasado que sólo puede escenificar la oportunidad siempre ya perdida de un mundo que podría haber sido $^{2}$ - tal como el amor por la "pasante", por ejemplo (Baudelaire, 1975, I, pp. 92-93) -, al mismo tiempo en que indica el eterno y "triunfal" retorno de los "vencedores" con sus "bienes culturales" y su "barbarie" (Benjamin, 2000, vol. 3, pp. 432-433), para evocar los términos célebres de la séptima tesis de Benjamin sobre la historia.

Es, por lo tanto, en esa paradójica disyunción temporal que vive el poeta-jugador moderno, entre la prisa de alcanzar, al fin, el fin del fin, y así - ¿quién sabe? - su vida póstuma, y la inquietud crítica de retener y desplegar la intensidad - la infinitud - potencial de lo que corre irremediablemente hacia su pérdida, pero que no deja de traer en sí una profecía de futuro - futuro que siempre se extrae bajo el signo del futuro compuesto de pretérito, único tiempo en que lo que acabó podria, al fin, haber tenido lugar. ${ }^{3}$

\footnotetext{
2 Descifrar clarividente que solo tiene lugar a posteriori, en el recuerdo, como dice Benjamin un poco después en el mismo texto: "¿No te hace señas, por entre las llamas, la noche anterior, cuyo lenguaje sólo ahora entiendes? [...] Como rayos ultravioleta, el recuerdo le muestra a cada uno, en el libro de la vida, una escrita que, invisible, en la condición de profecía, glosaba el texto.” (Benjamin, 1994, p. 64)

${ }^{3}$ Esto es puesto en evidencia al final del poema "À une passante" (Baudelaire, 1975, I, pp. 92-93).
} 
Pero hagamos ahora un salto y retornemos al presente, a nuestro presente histórico. Como decíamos al comienzo, lo que nos trae a lo que hoy llamamos realidad y a la experiencia de su transformación permanente pasa por la multiplicación exponencial de las redes de variables y de determinaciones que se ofrecen a la percepción, por la aceleración inusitada de las transformaciones y de los modos de atención, y de sus accidentes, claro está, lo que retira definitivamente de todo lenguaje y de toda figuración de realidad cualquier perspectiva de estabilidad. Si las posibilidades de soñar otros mundos se multiplicaron de tal modo en el tiempo de vida - o de muerte - que no podemos no proyectar hacia adelante, el fin de un mundo - o, para muchos, del mundo - se volvió, definitivamente, una sonora parte de la vida cotidiana.

Es de ese modo que, como en el comienzo del siglo XIX, el espectador de las fantasmagorías de Etienne Robertson - el primero gran "fantasmagorista" -, siempre perdido por entre el humo que lo separaba de cualquier certeza, vivimos sin siquiera saber lo que encontraremos o nos encontrará en la virtualidad sin fin de las pantallas que median nuestra relación con el cotidiano.

Si la "droga" por venir, si el "paraíso artificial" que se nos puede - siempre provisoriamente - proveer es remedio o veneno, no lo sabemos. "¿Qué importa?", habría dicho Baudelaire. De todos modos, según la concepción de experiencia de realidad que tengamos, si seguimos prefiriendo "el dolor a la muerte" y "el infierno a la nada", ¿qué comienzo, o recomienzo, es posible sino el olvido provisorio de la "ruina absoluta" que irremediablemente nos acecha, y la apuesta a $N^{\circ} 8$. Primer Semestre de 2018 
ciegas del jugador dispuesto a esquivar o, al menos a postergar, esa ruina? "Con los dedos rebuscando en el bolsillo vacío", como los jugadores del poema de Baudelaire, precisamos también siempre recomenzar para intentar, en un "cuerpo a cuerpo con el destino", "salvar lo que está perdido", como decía Benjamin (Benjamin, 2006, p.539), o, en otros términos, para darnos la chance de reencontrar, o de infinitizar, lo que, en cualquier parte del mundo o de los mundos en que vivimos, no cesa de perderse, y de no perderse, de acabarse, y de no acabarse, indecidiblemente...

\section{Referencias Bibliográficas}

Agamben, G. (1998). Stanze. Parole et fantasme dans la culture occidentale. Trad. de Yves Hersant. París: Payot \& Rivages.

Baudelaire, C. (1975-1976). Euvres complètes. Bibliothèque de la Pléiade. Texte établi, présenté et annoté par Claude Pichois. París: Gallimard, 2 vol.

---. (1973) Correspondance. Bibliothèque de la Pléiade. Texte établi, présenté et annoté par Claude Pichois. París: Gallimard, 2 vol.

Benjamin, W. (1989). Charles Baudelaire: um lírico no auge do capitalismo. Obras Escolhidas III. Trad. de José Carlos Martins Barbosa e Hemerson Alves Baptista. Río de Janeiro: Brasiliense.

---. (1994). Rua de mão única. Obras Escolhidas II. Trad. de José Carlos Martins Barbosa e Rubens Rodrigues Torres Filho. Río de Janeiro: Brasiliense, 4a ed. 
---. (2000). Euvres. Traduction de Maurice de Gandillac, revue par Rainer Rochlitz. París: Gallimard, 3 vol.

---. (2006). Passagens. Organização da edição brasileira de Willi Bolle. Colaboração de Olgária Matos. Trad. de Irene Aron e Cleonice Paes Barreto. Belo Horizonte: Editora UFMG; São Paulo: Imprensa Oficial do Estado de São Paulo.

---. (2013). Baudelaire. Edition établie par G. Agamben, Barbara Chitussi et Clemens-Carl Härle. Trad. de Patrick Charbonneau. París: La Fabrique.

Deguy, M. (2009). La fin dans le monde. París: Hermann.

Guyaux, A. (2007). Baudelaire. Un demi-siècle de lectures des Fleurs du mal (1855-1905). París: Presses universitaires de Paris-Sorbonne, coll. «Mémoire de la critique».

Hirt, A. (1998). Baudelaire. L'exposition de la poésie. París: Éditions Kimé.

---. (2010). Baudelaire. Le monde va finir. París: Editions Kimé. 